\title{
Dense coding capacity of a quantum channel
}

\author{
Riccardo Laurenza, ${ }^{1}$ Cosmo Lupo, ${ }^{2}$ Seth Lloyd ${ }^{3,4}$ and Stefano Pirandola ${ }^{4,5}$ \\ ${ }^{1}$ QSTAR, INO-CNR and LENS, Largo Enrico Fermi 2, 50125 Firenze, Italy \\ ${ }^{2}$ Department of Physics and Astronomy, University of Sheffield, Hounsfield Road, Sheffield S3 7RH, United Kingdom \\ ${ }^{3}$ Department of Mechanical Engineering, Massachusetts Institute of Technology, Cambridge, Massachusetts 02139, USA \\ ${ }^{4}$ Research Laboratory of Electronics, Massachusetts Institute of Technology, Cambridge, Massachusetts 02139, USA \\ ${ }^{5}$ Department of Computer Science, University of York, York YO10 5GH, United Kingdom
}

(Received 20 March 2019; accepted 13 January 2020; published 9 April 2020)

\begin{abstract}
We consider the fundamental protocol of dense coding of classical information assuming that noise affects both the forward and backward communication lines between Alice and Bob. Assuming that this noise is described by the same quantum channel, we define its dense coding capacity by optimizing over all adaptive strategies that Alice can implement, while Bob encodes the information by means of Pauli operators. Exploiting techniques of channel simulation and protocol stretching, we are able to establish the dense coding capacity of Pauli channels in arbitrary finite dimension, with simple formulas for depolarizing and dephasing qubit channels.
\end{abstract}

DOI: 10.1103/PhysRevResearch.2.023023

\section{INTRODUCTION}

Dense coding, also known as superdense coding, has been one of the first examples of how quantum entanglement can boost information and communication technology [1]. Now recognized as an essential resource for quantum communication and information processing [2-5], quantum entanglement describes correlations outside the classical realm and it is at the core of the realization of many methods, including quantum teleportation [6,7], quantum cryptography [8-10], boson sampling [11,12], and random circuit sampling [13,14]. The dense coding protocol allows two parties to transmit classical information encoded on quantum systems with the aid of shared entanglement. By employing a bipartite entangled state, it is possible to encode $2 \log _{2} d$ bits of classical information in a $d$-dimensional system, thus overcoming the upper bound $\log _{2} d$ on the unassisted classical capacity.

In ideal conditions, a dense coding scheme exploits a noiseless quantum channel between Alice and Bob. Through this quantum channel, Alice sends to Bob part $B$ of a bipartite entangled state $\sigma_{A B}$. Once received by Bob, system $B$ is subject to a Pauli operator $U_{x}$ with probability $P_{x}$. The encoded system is sent back to Alice through the second use of the noiseless quantum channel. At the output, Alice implements a joint quantum measurement on $A$ and $B$ to retrieve the classical information. In this case, the capacity $C\left(\sigma_{A B}\right)$ is $[15,16]$

$$
C\left(\sigma_{A B}\right)=\max \left\{\log _{2} d, \log _{2} d+S\left(\sigma_{B}\right)-S\left(\sigma_{A B}\right)\right\},
$$

where $\sigma_{B}=\operatorname{Tr}_{A} \sigma_{A B}$ and $S(\sigma):=-\operatorname{Tr}\left(\sigma \log _{2} \sigma\right)$ is the von Neumann entropy [17]. For a maximally entangled resource state $\sigma_{A B}$ one has $C=2 \log _{2} d$.

Published by the American Physical Society under the terms of the Creative Commons Attribution 4.0 International license. Further distribution of this work must maintain attribution to the author(s) and the published article's title, journal citation, and DOI.
In a realistic scenario, noise must be explicitly included in the protocol. For instance, noise can affect the transmission of quantum systems from the sender (Bob) to the receiver (Alice), after the entangled resource state has been perfectly distributed. This is the typical scenario in the definition of entanglement-assisted protocols whose capacity is known $[18,19]$. More realistically, noise may also affect the distribution itself of the resource state from Alice to Bob. This scenario has been previously studied in Refs. [20-22] where it has been called "two-sided" noisy dense coding but no capacity has been established.

This is the aim of this paper where the two-sided protocol is formulated in a general feedback-assisted fashion. Here the round-trip transmission of the quantum systems between Alice and Bob is interleaved by two adaptive quantum operations (QOs) performed by Alice, which are optimized and updated on the basis of the previous rounds. At the same time, Bob may also optimize his classical encoding strategy, i.e., the probability distribution of his Pauli encoders. Optimizing over these protocols we define the dense coding capacity of a quantum channel between Alice and Bob. We then use simulation techniques [23-26] that allow us to simplify the structure of the protocol and derive a single-letter upper bound for this capacity. This quantity is explicitly computed for a Pauli channel in arbitrary $d$ dimension, with remarkably simple formulas for qubit channels, such as the depolarizing and the dephasing channel.

\section{DENSE CODING PROTOCOL}

Let us recall the expressions of Pauli operators in a $d$ dimensional Hilbert space. On a computational basis $\{|j\rangle\}$, we may define the two shift operators

$$
X|j\rangle=|j \oplus 1\rangle, Z|j\rangle=\omega^{j}|j\rangle,
$$

where $\oplus$ is modulo $d$ addition and $\omega:=\exp (2 i \pi / d)$. We may then consider the $d^{2}$ Pauli operators $X^{l} Z^{m}$ that, for simplicity, we denote by $U_{x}$ with collapsed index $x=l, m$. For $d=2$, 


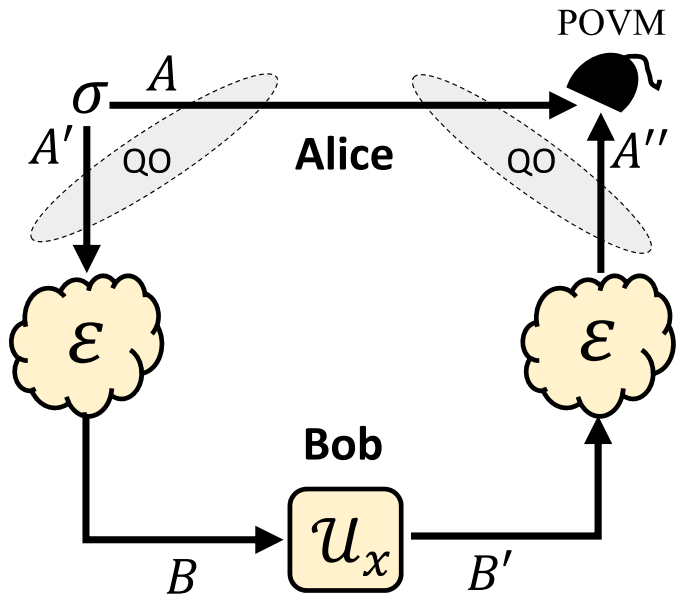

FIG. 1. Two-sided noisy dense coding over a quantum channel $\mathcal{E}$. Alice prepares locally the bipartite state $\sigma_{A A^{\prime}}$ and sends system $A^{\prime}$ to Bob who performs the Pauli unitary encoding $\mathcal{U}_{x}$ and sends the system back through the quantum channel. At the output Alice performs a joint positive-valued operator measure (POVM) in order to retrieve $x$. In an adaptive version of the protocol, Alice performs quantum operations (QOs) on her input and output systems which are generally updated and optimized round by round. These QOs may also be conditioned by an extra assisting variable which is communicated back by Bob.

these operators provide the standard qubit Pauli operators $X$, $Y, Z$ plus the identity $I$. In the following we use the compact notation $\mathcal{U}_{x}(\rho):=U_{x} \rho U_{x}^{\dagger}$.

Now consider the scheme depicted in Fig. 1 where the communication line between Alice and Bob is affected by a completely positive trace-preserving $(\mathrm{CPTP})$ map $\mathcal{E}$. Alice's resource state $\sigma_{A A^{\prime}}$ is defined on a $d \times d$-dimensional Hilbert space. Part $A^{\prime}$ is sent to Bob who encodes classical variable $X:=\left\{x, \pi_{x}\right\}$ by means of $d^{2}$ Pauli operators $\mathcal{U}_{x}$ which are chosen with probability $\pi_{x}$. In this way, Bob generates the state

$$
\sigma_{A B^{\prime}}(x):=\left[\mathcal{I}_{A} \otimes\left(\mathcal{U}_{x} \circ \mathcal{E}\right)_{A^{\prime}}\right]\left(\sigma_{A A^{\prime}}\right),
$$

where $\mathcal{I}(\rho):=I \rho I^{\dagger}$ is the identity map. Once system $B^{\prime}$ is sent back through the channel, Alice receives the output system $A^{\prime \prime}$ in the state $\rho_{A A^{\prime \prime}}(x):=\left(\mathcal{I}_{A} \otimes \mathcal{E}_{x}\right)\left(\sigma_{A A^{\prime}}\right)$ where we have defined the encoding channel

$$
\mathcal{E}_{x}:=\mathcal{E} \circ \mathcal{U}_{x} \circ \mathcal{E} .
$$

In order to retrieve the value of $x$, Alice performs a joint quantum measurement on $A$ and $A^{\prime \prime}$. Asymptotically (i.e., for many repetitions of the protocol), the accessible information of Alice's output ensemble $\left\{\pi_{x}, \rho_{A A^{\prime \prime}}(x)\right\}$ is given by the Holevo bound [27],

$$
\chi\left(\left\{\pi_{x}, \rho_{A A^{\prime \prime}}(x)\right\}\right)=S\left[\sum_{x} \pi_{x} \rho_{A A^{\prime \prime}}(x)\right]-\sum_{x} \pi_{x} S\left[\rho_{A A^{\prime \prime}}(x)\right] .
$$

The one-shot dense coding capacity (1-DCC) of the channel $C_{D}^{(1)}(\mathcal{E})$ is obtained by optimizing over Bob's encoding vari-

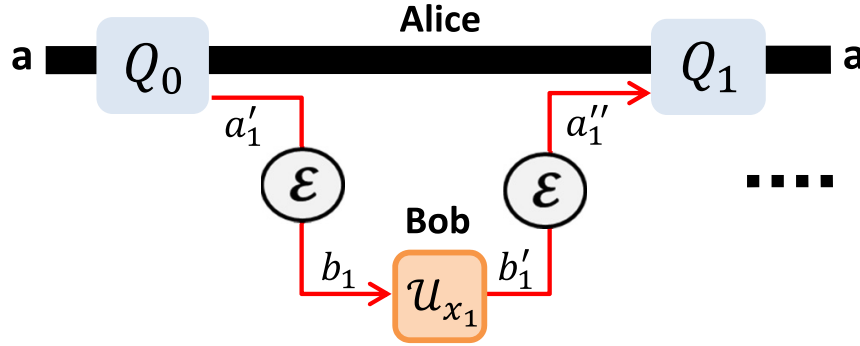

FIG. 2. Adaptive dense coding protocol over the quantum channel $\mathcal{E}$. Each encoding and round-trip transmission occurs between two quantum operations (QOs) which are applied to Alice's local register of quantum systems (e.g., this register can be thought to be part of a quantum computer). In the figure, we show the first use of the protocol, where Alice picks a quantum system $a_{1}^{\prime}$ from her register, and she sends it to Bob through the noisy channel $\mathcal{E}$. Once the output $b_{1}$ is received, Bob applies a local Pauli operator $\mathcal{U}_{x_{1}}$ encoding letter $x_{1}$ with probability $\pi_{x_{1}}$. The encoded system $b_{1}^{\prime}$ is then sent back to Alice through the backward use of the channel $\mathcal{E}$, with the output system $a_{1}^{\prime \prime}$ becoming part of Alice's local register.

able and Alice's input source, i.e., we may write

$$
C_{D}^{(1)}(\mathcal{E})=\max _{\sigma, \pi_{x}} \chi\left[\left\{\pi_{x}, \rho_{A A^{\prime \prime}}(x)\right\}\right] .
$$

\section{ADAPTIVE DENSE CODING}

Consider now dense coding over a quantum channel $\mathcal{E}$ where Alice performs QOs in an adaptive fashion. Alice has a quantum register $\mathbf{a}$ as in Fig. 2. This is an ensemble of $d$-dimensional quantum systems that she can manipulate and use for the quantum communication. At the beginning Alice performs a QO $Q_{0}$ on her register a in order to prepare it in some initial state $\rho_{\mathbf{a}}^{0}$. She then selects one system $a_{1}^{\prime} \in \mathbf{a}$ and sends it through the quantum channel $\mathcal{E}$. Once Bob receives the corresponding output system $b_{1}$, he encodes the letter $x_{1}$ by applying a Pauli operator $\mathcal{U}_{x_{1}}$ with probability $\pi_{x_{1}}$. This procedure gives rise to the state $\rho_{\mathbf{a} b_{1}^{\prime}}^{0}\left(x_{1}\right)$. Bob sends the system $b_{1}^{\prime}$ backward to Alice through $\mathcal{E}$. At the output, Alice incorporates the received system $a_{1}^{\prime \prime}$ in her local register which is updated as $\mathbf{a} a_{1}^{\prime \prime} \rightarrow \mathbf{a}$. Next, Alice performs an optimized QO $Q_{1}$ on the register with output state $\rho_{\mathbf{a}}^{1}\left(x_{1}\right)$. In the second transmission Alice picks another system $a_{2}^{\prime} \in \mathbf{a}$ and she transmits it to Bob who receives $b_{2}$. Bob applies the second Pauli operator $\mathcal{U}_{x_{2}}$ with probability $\pi_{x_{2} \mid x_{1}}$ and sends the system back to Alice who performs another optimized QO $Q_{2}$ obtaining the state $\rho_{\mathbf{a}}^{2}\left(x_{1} x_{2}\right)$. After $n$ uses, Alice's output state will be $\rho_{\mathbf{a}}^{n}\left(\mathbf{x}_{n}\right)$ where $\mathbf{x}_{i}=x_{1} x_{2} \ldots x_{i}$ is the encoded message with probability $\pi_{\mathbf{x}_{i}}=\pi_{x_{i} \mid x_{i-1} \ldots x_{1}} \ldots \pi_{x_{2} \mid x_{1}} \pi_{x_{1}}$.

On average, Alice receives the ensemble $\left\{\pi_{\mathbf{x}_{n}}, \rho_{\mathbf{a}}^{n}\left(\mathbf{x}_{n}\right)\right\}$ where the output state $\rho_{\mathbf{a}}^{n}\left(\mathbf{x}_{n}\right)$ depends on the encoded classical information and the sequence of QOs $\mathcal{Q}:=$ $\left\{Q_{1}, Q_{2}, \ldots Q_{n}\right\}$. Alice's deferred measurement [2] will be done on the final state. For large $n$, and optimizing the Holevo information of the ensemble $\mathcal{P}$ over all the possible sequences $\mathcal{Q}$, we define the dense coding capacity (DCC) of the quantum channel $\mathcal{E}$ as

$$
C_{D}(\mathcal{E}):=\sup _{\mathcal{Q}} \max _{\pi_{\mathbf{x}_{n}}} \lim _{n} n^{-1} \chi\left[\left\{\pi_{\mathbf{x}_{n}}, \rho_{\mathbf{a}}^{n}\left(\mathbf{x}_{n}\right)\right\}\right] .
$$


(a)

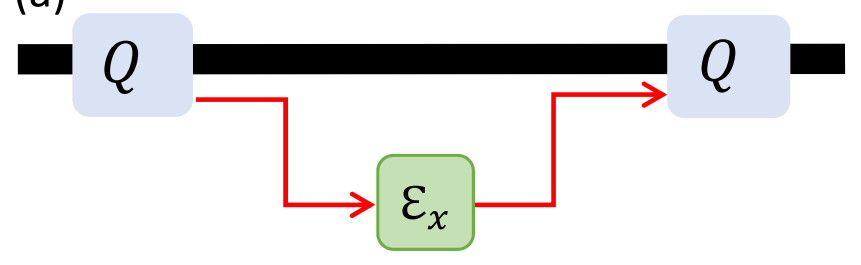

(b)

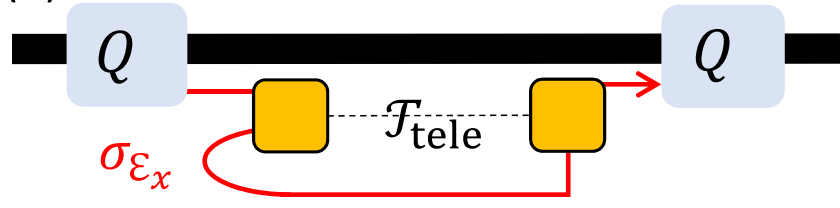

(c)

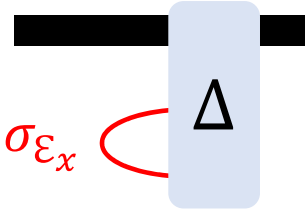

(d)

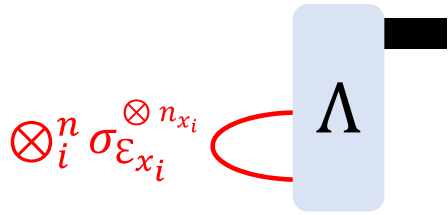

FIG. 3. Stretching of adaptive dense coding. (a) Replace the round-trip process of Fig. 2 with a total $x$-depending channel $\mathcal{E}_{x}$, following Eq. (4). (b) Now simulate the total channel $\mathcal{E}_{x}$ by teleporting $\mathcal{T}_{\text {tele }}$ over the Choi matrix $\sigma_{\mathcal{E}_{x}}$, i.e., we make use of $\mathcal{T}_{\text {tele }}\left(\rho \otimes \sigma_{\mathcal{E}_{x}}\right)$. (c) Collapse the QOs and the simulation LOCC $\mathcal{T}_{\text {tele }}$ into a single QO $\Delta$ so that the Choi matrix $\sigma_{\mathcal{E}_{x}}$ can be stretched out of the adaptive operation. (d) Repeat for all the $n$ uses, so as to collect an ensemble of Choi matrices subject to a global QO $\Lambda$.

Note that this definition is more general than a regularized version $C_{D}^{\infty}(\mathcal{E})$ of Eq. (6), where Alice prepares a large multipartite input state, sends part of this state through $n$ uses of the round trip, and then performs a global measurement of the total output. In fact, Eq. (7) assumes that Alice's input can also be updated round by round on the basis of feedback from Bob [28].

\section{SINGLE-LETTER UPPER BOUND}

We now exploit a number of ingredients from recent literature to derive a computable upper bound for the DCC. Recall that, for any finite-dimensional quantum channel $\mathcal{E}$, we may write the simulation $\mathcal{E}(\rho)=\mathcal{T}(\rho \otimes \sigma)$, where $\mathcal{T}$ is a trace-preserving LOCC and $\sigma$ a resource state [23]. Furthermore, suppose that the channel is covariant with respect to Pauli operators so that, for any Pauli $\mathcal{U}$, we may write $\mathcal{E} \circ \mathcal{U}=\mathcal{U}^{\prime} \circ \mathcal{E}$ for some generally different Pauli $\mathcal{U}^{\prime}$. In this case the channel is Pauli covariant and we may write $[23,25]$ $\mathcal{E}(\rho)=\mathcal{T}_{\text {tele }}\left(\rho \otimes \sigma_{\mathcal{E}}\right)$, where $\mathcal{T}_{\text {tele }}$ is a teleportation LOCC and $\sigma_{\mathcal{E}}$ is the channel's Choi matrix, i.e., $\sigma_{\mathcal{E}}:=\mathcal{I}_{A} \otimes \mathcal{E}\left(\Phi_{A B}\right)$ with $\Phi_{A B}$ being a maximally entangled state. Note that the Pauli unitaries $\mathcal{U}_{x}$ are jointly Pauli covariant, i.e., we may certainly write $\mathcal{U}_{x} \circ \mathcal{U}=\mathcal{U}^{\prime} \circ \mathcal{U}_{x}$ where $\mathcal{U}^{\prime}$ is the same for any $x$ (since a Pauli operator either commutes or anticommutes with another Pauli operator). Therefore, if $\mathcal{E}$ is Pauli covariant, we also have that the encoding channel $\mathcal{E}_{x}$ is jointly Pauli covariant. We may therefore write the channel simulation $\mathcal{E}_{x}(\rho)=\mathcal{T}_{\text {tele }}\left(\rho \otimes \sigma_{\mathcal{E}_{x}}\right)$ in terms of its Choi matrix.

$$
\sigma_{\mathcal{E}_{x}}:=\mathcal{I}_{A} \otimes \mathcal{E}_{x}\left(\Phi_{A B}\right) .
$$

The next step is the stretching of the protocol as represented in Fig. 3 and explained in the figure caption. Thanks to this procedure the output state can be decomposed in a tensor product of Choi matrices up to a global QO $\Lambda$, i.e., we may write

$$
\rho_{\mathbf{a}}^{n}\left(\mathbf{x}_{n}\right)=\Lambda\left(\sigma_{\mathcal{E}_{x_{1}}}^{\otimes n_{x_{1}}} \otimes \sigma_{\mathcal{E}_{x_{2}}}^{\otimes n_{x_{2}}} \otimes \cdots \otimes \sigma_{\mathcal{E}_{x_{n}}}^{\otimes n_{x_{n}}}\right),
$$

where the $n_{x_{i}}$ is the number of $x_{i}$ occurrences in the message $\mathbf{x}_{n}$. This is given by $n_{x_{i}}=n \pi_{x_{i}}$ where $\pi_{x_{i}}=\sum_{j \neq i} \pi_{\mathbf{x}_{n}}$ is the marginal probability. Thanks to Eq. (9) we can simplify the Holevo quantity in Eq. (7). In fact, by using ( $\star$ ) the contractivity under CPTP maps of the Holevo quantity, and $(\bullet)$ the subadditivity of the von Neumann entropy $S$ under tensor products, we may write

$$
\begin{aligned}
\chi & {\left[\left\{\pi_{\mathbf{x}_{n}}, \rho_{\mathbf{a}}^{n}\left(\mathbf{x}_{n}\right)\right\}\right] \stackrel{(\star)}{\leqslant} \chi\left(\left\{\pi_{\mathbf{x}_{n}}, \bigotimes_{i=1}^{n} \sigma_{\mathcal{E}_{x_{i}}}^{\otimes n_{x_{i}}}\right\}\right) } \\
= & S\left(\sum_{\mathbf{x}_{n}} \pi_{\mathbf{x}_{n}} \bigotimes_{i} \sigma_{\mathcal{E}_{x_{i}}}^{\otimes n_{x_{i}}}\right)-\sum_{\mathbf{x}_{n}} \pi_{\mathbf{x}_{n}} S\left(\bigotimes_{i} \sigma_{\mathcal{E}_{x_{i}}}^{\otimes n_{x_{i}}}\right) \\
& \stackrel{(\bullet)}{\leqslant} n_{x_{1}} S\left(\sum_{\mathbf{x}_{n}} \pi_{\mathbf{x}_{n}} \sigma_{\mathcal{E}_{x_{1}}}\right)+\cdots+n_{x_{n}} S\left(\sum_{\mathbf{x}_{n}} \pi_{\mathbf{x}_{n}} \sigma_{\mathcal{E}_{x_{n}}}\right) \\
& -n_{x_{1}} \sum_{\mathbf{x}_{n}} \pi_{\mathbf{x}_{n}} S\left(\sigma_{\mathcal{E}_{x_{1}}}\right)-\cdots-n_{x_{n}} \sum_{\mathbf{x}_{n}} \pi_{\mathbf{x}_{n}} S\left(\sigma_{\mathcal{E}_{x_{n}}}\right) \\
\leqslant & n S\left(\sum_{x} \pi_{x} \sigma_{\mathcal{E}_{x}}\right)-n \sum_{x} \pi_{x} S\left(\sigma_{\mathcal{E}_{x}}\right) \\
= & n \chi\left(\left\{\pi_{x}, \sigma_{\mathcal{E}_{x}}\right\}\right),
\end{aligned}
$$

where $\pi_{x}$ is the marginal probability of a generic letter $x$ and the Choi matrix $\sigma_{\mathcal{E}_{x}}$ is defined in Eq. (8). Note that, in the last inequality of Eq. (10), we also use the fact that a random code $[2,29]$ is known to achieve the Holevo bound for discrete memoryless quantum channels [30,31].

By using Eq. (10) in the definition of Eq. (7), we may then get rid of the supremum over $\mathcal{Q}$ and the asymptotic limit in $n$. We may therefore write a single-letter upper bound for the DCC of a Pauli-covariant channel $\mathcal{E}$ as

$$
C_{D}(\mathcal{E}) \leqslant \max _{\pi_{x}} \chi\left(\left\{\pi_{x}, \sigma_{\mathcal{E}_{x}}\right\}\right),
$$

where $\pi_{x}$ is the marginal probability distribution of Bob's encoding variable, and $\sigma_{\mathcal{E}_{x}}$ is the Choi matrix of the encoding channel $\mathcal{E}_{x}$ in Eq. (4). Note that the upper bound in Eq. (11) may be reached asymptotically by a nonadaptive protocol where Alice prepares maximally entangled states $\Phi_{A A^{\prime}}$ and sends $A^{\prime}$ through the channel, while Bob applies independent Pauli operators $\mathcal{U}_{x}$ with optimized probability $\pi_{x}$. Therefore, for a Pauli-covariant channel we conclude that

$$
C_{D}(\mathcal{E})=C_{D}^{(1)}(\mathcal{E})=\max _{\pi_{x}} \chi\left(\left\{\pi_{x}, \sigma_{\mathcal{E}_{x}}\right\}\right) .
$$

Remarkably, no adaptiveness or regularization is needed to achieve the best possible dense coding performance with a Pauli-covariant channel. 
Before we proceed, some observations are in order. First of all, let us note that the condition of Pauli covariance allows us to reduce the (adaptive) dense coding capacity of a quantum channel $\mathcal{E}$ (Fig. 2) to the quantum reading capacity [32] of a corresponding ensemble of encoding channels $\mathcal{E}_{x}:=\mathcal{E} \circ$ $\mathcal{U}_{x} \circ \mathcal{E}$ [Fig. 3(a)]. This interesting connection is discussed in more detail in Ref. [33]. Then, let us note that, in a more general scenario, the forward channel from $A^{\prime}$ to $B$ and the backward channel from $B^{\prime}$ to $A^{\prime \prime}$ (Fig. 1) may be described by two different completely positive maps, say $\mathcal{E}_{1}$ and $\mathcal{E}_{2}$ respectively. We can easily extend Eq. (7) to define the dense coding capacity of the composition $\mathcal{E}_{2} \circ \mathcal{E}_{1}$. Suppose now that both $\mathcal{E}_{1}$ and $\mathcal{E}_{2}$ are Pauli covariant. Then, via Eq. (4), we can define a new encoding channel $\mathcal{E}_{x}^{1,2}:=\mathcal{E}_{2} \circ \mathcal{U}_{x} \circ \mathcal{E}_{1}$ and repeat the above stretching technique to obtain a single letter formula for $C_{D}\left(\mathcal{E}_{2} \circ \mathcal{E}_{1}\right)$ in terms of the Choi matrix $\sigma_{\mathcal{E}_{1}^{1,2}}$ of the encoding channel $\mathcal{E}_{x}^{1,2}$. This procedure extends Eq. (12) to the formula $C_{D}\left(\mathcal{E}_{2} \circ \mathcal{E}_{1}\right)=\max _{\pi_{x}} \chi\left(\left\{\pi_{x}, \sigma_{\mathcal{E}_{x}^{1,2}}\right\}\right)$.

\section{DENSE CODING CAPACITY OF PAULI CHANNELS}

The main result in Eq. (12) can be applied to any Pauli channel at any finite dimension $d$. For any $d \geqslant 2$, a Pauli channel takes the form

$$
\mathcal{E}^{d}(\rho)=\sum_{k, r=0}^{d-1} p_{k r}\left(X^{k} Z^{r}\right) \rho\left(X^{k} Z^{r}\right)^{\dagger},
$$

where $p_{k r}$ is a probability distribution, and $X$ and $Z$ are the $d$-dimensional shift operators in Eq. (2). For this channel, we may easily write an explicit formula for its DCC capacity. In evaluating the Holevo bound, we notice that von Neumann entropy $S\left(\sum_{x} \pi_{x} \sigma_{\mathcal{E}_{x}^{d}}\right)$ is maximized by the uniform probability $\pi_{x}=1 / d^{2}$ and we can write $S\left(\sum_{x} \pi_{x} \sigma_{\mathcal{E}_{x}^{d}}\right)=\log _{2} d^{2}$. Then, using the invariance of the entropy under unitary transformations, one has $\sum_{x} \pi_{x} S\left(\sigma_{\mathcal{E}_{x}^{d}}\right)=S\left[\mathcal{I}_{A} \otimes \mathcal{E}^{d}\left(\sigma_{\mathcal{E}^{d}}\right)\right]$. Therefore, for the Holevo quantity in Eq. (12) we may write

$$
C_{D}\left(\mathcal{E}^{d}\right)=\log _{2} d^{2}-S\left[\mathcal{I}_{A} \otimes \mathcal{E}^{d}\left(\sigma_{\mathcal{E}^{d}}\right)\right] .
$$

As expected this is strictly less than the entanglement-assisted classical capacity of the channel, given by $[18,19]$

$$
C_{E}(\mathcal{E})=\log _{2} d^{2}-S\left(\sigma_{\mathcal{E}^{d}}\right) .
$$

Consider a qubit depolarizing channel, which is a Pauli channel of the form

$$
\mathcal{E}_{\text {depol }}^{2}(\rho)=\left(1-\frac{3}{4} p\right) \rho+\frac{p}{4}(X \rho X+Y \rho Y+Z \rho Z),
$$

for some probability $p$. Then, it is straightforward to see that $C_{D}\left(\mathcal{E}_{\text {depol }}^{2}\right)=2-h_{2}(\alpha)-\alpha \log _{2} 3$, where $h_{2}(x)=$ $-x \log _{2} x-(1-x) \log _{2}(1-x)$ is the binary entropy function and $\alpha:=3 / 4 p(2-p)$. Then, consider a qubit dephasing channel, which takes the form

$$
\mathcal{E}_{\text {deph }}^{2}(\rho)=(1-p) \rho+p Z \rho Z .
$$

Its DCC is equal to the following expression:

$$
C_{D}\left(\mathcal{E}_{\text {deph }}^{2}\right)=2\left[1-h_{2}(p)\right],
$$

for $p \leqslant 1 / 2$ and zero otherwise.

\section{CONCLUSION}

In this work we have considered the most general adaptive protocol for the dense coding of classical information in a realistic scenario where noise affects both the communication lines between Alice and Bob. Assuming that this noise is modelled by the same quantum channel, we define its dense coding capacity as the maximum amount of classical information (per round-trip use) that Bob can transmit to Alice. We assume that Bob is implementing Pauli encoders with an optimized probability distribution and Alice is using quantum registers that are adaptively updated and optimized in the process. For the Pauli-covariant channel, we find that this capacity reduces to a single-letter version based on a protocol which is nonadaptive and one-shot (i.e., using iid input states). In particular, we can establish exact formulas for the dense coding capacity of Pauli channels.

Note that our approach departs from the definition of entanglement-assisted classical capacity of a quantum channel $[18,19]$, where it is implicitly required that the parties either have a noiseless side quantum channel for distributing entangled sources or they have previously met and stored quantum entanglement in ideal long-life quantum memories. Our treatment and definition of dense coding capacity removes these assumptions assuming that the entanglement source is itself distributed through the noisy channel and, therefore, it is realistically degraded by the environment. Because of this feature, our capacity can also be seen as an upper bound for the key rates of two-way quantum key distribution protocols that are related to the dense coding idea [34-38].

\section{ACKNOWLEDGMENTS}

This work was supported by the EPSRC via the "UK Quantum Communications Hub" (Grant No. EP/T001011/1) and the European Commission via "Continuous Variable Quantum Communications” (CiViQ, Grant No. 820466).
[1] C. H. Bennett and S. J. Wiesner, Phys. Rev. Lett. 69, 2881 (1992).

[2] M. A. Nielsen and I. L. Chuang, Quantum Computation and Quantum Information (Cambridge University Press, Cambridge, UK, 2000).

[3] J. Watrous, The Theory of Quantum Information (Cambridge University Press, Cambridge, UK, 2018).
[4] A. Holevo, Quantum Systems, Channels, Information: A Mathematical Introduction (De Gruyter, Berlin, 2012).

[5] C. Weedbrook, S. Pirandola, R. Garcia-Patrón, N. J. Cerf, T. C. Ralph, J. H. Shapiro, and S. Lloyd, Rev. Mod. Phys. 84, 621 (2012).

[6] C. H. Bennett, G. Brassard, C. Crepeau, R. Jozsa, A. Peres, and W. K. Wootters, Phys. Rev. Lett. 70, 1895 (1993). 
[7] S. Pirandola, J. Eisert, C. Weedbrook, A. Furusawa, and S. L. Braunstein, Nat. Photonics 9, 641 (2015).

[8] C. H. Bennett, and G. Brassard, Proc. IEEE Int. Conf. Comput., Syst. Signal Process. 175, 8 (1984).

[9] A. K. Ekert, Phys. Rev. Lett. 67, 661 (1991).

[10] S. Pirandola, U. L. Andersen, L. Banchi, M. Berta, D. Bunandar, R. Colbeck, D. Englund, T. Gehring, C. Lupo, C. Ottaviani, J. Pereira, M. Razavi, J. S. Shaari, M. Tomamichel, V. C. Usenko, G. Vallone, P. Villoresi, and P. Wallden, arXiv:1906.01645.

[11] S. Aaronson and A. Arkhipov, Theory Comput. 9, 143 (2013).

[12] H. Wang, J. Qin, X. Ding, M.-C. Chen, S. Chen, X. You, Y.M. He, X. Jiang, L. You, Z. Wang et al., Phys. Rev. Lett. 123, 250503 (2019).

[13] A. Bouland, B. Fefferman, C. Nirkhe, and U. Vazirani, arXiv:1803.04402.

[14] F. Arute et al., Nature (London) 574, 505 (2019).

[15] T. Hiroshima, J. Phys. A 34, 6907 (2001).

[16] M. Ziman and V. Bužek, Phys. Rev. A 67, 042321 (2003).

[17] It is clear that entangled states with positive partial transpose, i.e., bound entangled states, are not useful for dense coding, since for these states we have $S\left(\sigma_{B}\right)-S\left(\sigma_{A B}\right)<0$ so that they cannot be exploited to transmit at a rate greater than $\log _{2} d$.

[18] C. H. Bennett, P. W. Shor, J. A. Smolin, and A. V. Thapliyal, Phys. Rev. Lett. 83, 3081 (1999)

[19] C. H. Bennett, P. W. Shor, J. A. Smolin, and A. V. Thapliyal, IEEE Trans. Inf. Theory 48, 2637 (2002).

[20] Z. Shadman, H. Kampermann, C. Macchiavello, and D. Bruß, New J. Phys. 12, 073042 (2010).

[21] Z. Shadman, H. Kampermann, D. Bruß, and C. Macchiavello, Phys. Rev. A 84, 042309 (2011).

[22] Z. Shadman, H. Kampermann, C. Macchiavello, and D. Bruß, Quantum Meas. Quantum Metrol. 1, 21 (2013).
[23] S. Pirandola, R. Laurenza, C. Ottaviani, and L. Banchi, Nat. Commun. 8, 15043 (2017).

[24] S. Pirandola, B. Roy Bardhan, T. Gehring, C. Weedbrook, and S. Lloyd, Nat. Photonics 12, 724 (2018).

[25] S. Pirandola, S. L. Braunstein, R. Laurenza, C. Ottaviani, and L. Banchi, Quantum Sci. Technol. 3, 035009 (2018).

[26] R. Laurenza, C. Lupo, G. Spedalieri, S. L. Braunstein, and S. Pirandola, Quantum Meas. Quantum Metrol. 5, 1 (2018).

[27] A. S. Holevo, Prob. Inf. Transm. 9, 177 (1973).

[28] Note that one might consider a generalized dense coding protocol, where Bob uses arbitrary encoding unitaries instead of Pauli operators. Correspondingly, we may introduce a generalized dense coding capacity $\tilde{C}_{D}(\mathcal{E})$ as in Eq. (7) but replacing the maximization as $\max _{\pi_{\mathbf{x}_{n}}} \rightarrow \max _{\left\{\pi_{\mathbf{x}_{n}}, U_{\left.\mathbf{x}_{n}\right\}}\right\}}$, where $U_{\mathbf{x}_{n}}$ are sequences of arbitrary unitary encoders. By definition, we have $C_{D}(\mathcal{E}) \leqslant \tilde{C}_{D}(\mathcal{E})$.

[29] C. E. Shannon, Bell Syst. Tech. J. 27, 379 (1948).

[30] A. S. Holevo, IEEE Trans. Inf. Theory 44, 269 (1998).

[31] B. Schumacher and M. D. Westmoreland, Phys. Rev. A 56, 131 (1997).

[32] S. Pirandola, C. Lupo, V. Giovannetti, S. Mancini, and S. L. Braunstein, New J. Phys. 13, 113012 (2011).

[33] S. Pirandola, arXiv:1812.08810.

[34] K. Boström and T. Felbinger, Phys. Rev. Lett. 89, 187902 (2002).

[35] Q.-Y. Cai, Phys. Rev. Lett. 91, 109801 (2003).

[36] F.-G. Deng and G. L. Long, Phys. Rev. A 69, 052319 (2004).

[37] Q.-Y. Cai and B.-W. Li, Chin. Phys. Lett. 21, 601 (2004).

[38] M. Lucamarini and S. Mancini, Phys. Rev. Lett. 94, 140501 (2005). 